\title{
L'enseignement bilingue
}

\section{Françoise Profit}

\section{(2) OpenEdition}

Journals

Édition électronique

URL : https://journals.openedition.org/ries/1823

DOI : $10.4000 /$ ries. 1823

ISSN : 2261-4265

\section{Éditeur}

France Education international

\section{Édition imprimée}

Date de publication : 1 décembre 2002

Pagination : $\mathrm{fr}$

ISBN : 978-2854-2-0555-8

ISSN : 1254-4590

\section{Référence électronique}

Françoise Profit, «L'enseignement bilingue », Revue internationale d'éducation de Sèvres [En ligne], 31 | décembre 2002, mis en ligne le 24 novembre 2011, consulté le 06 juillet 2021. URL : http:// journals.openedition.org/ries/1823; DOI : https://doi.org/10.4000/ries.1823

Ce document a été généré automatiquement le 6 juillet 2021

(c) Tous droits réservés 


\title{
L'enseignement bilingue
}

\author{
Françoise Profit
}

\section{Programmes d'évaluation}

$1 \quad$ Les demandes d'évaluation des systèmes éducatifs émanent des pouvoirs politiques qui souhaitent y trouver une aide à la négociation, à la décision et à l'orientation de leurs politiques éducatives prises dans une tension entre mouvement de décentralisation et mondialisation. Les pouvoirs politiques prennent appui sur les études transnationales effectuées par des organismes internationaux, comme l'OCDE ou l'UNESCO, aux conclusions reconnues pour leur objectivité à «évaluer l'innovation et innover dans l'évaluation ${ }^{1} »$. S'inspirant d'une démarche qualité issue du monde de l'entreprise, ces organismes, à travers les évaluations effectuées, offrent aux différents pays la possibilité de la comparaison.

2 Nous nous intéressons ici aux évaluations issues des organismes internationaux en retenant une approche par les résultats, qui ne comporte pas de sites d'indicateurs. Enfin, nous terminons en prenant un seul exemple national, l'exemple français, qui couvre l'ensemble du domaine de l'évaluation. Dans un numéro ultérieur, nous aborderons l'évaluation des élèves et des enseignants au niveau international.

\section{L'évaluation des systèmes éducatifs internationaux}

\section{Terminologie}

3 Afin de cerner la terminologie d'un domaine aussi vaste que celui de l'évaluation, l'OCDE offre un glossaire trilingue (français, anglais, espagnol) des principaux termes relatifs à l'évaluation et à la gestion axée sur les résultats. Ce glossaire, au format pdf, permet de clarifier les concepts, d'harmoniser et d'affiner la terminologie spécifique au domaine. http://www.oecd.org/pdf/M00030000/M00030075.pdf/. 


\section{Sources}

\section{Base de données / ressources}

4 La base de données ERIC (Educational Resources Information Center) http://nces.ed.gov/ pubsearch/ fonctionne avec le site http://ericae.net/ qui propose une base de données bibliographiques, et un grand nombre de liens traitant de l'évaluation: http:// ericae.net (USA).

5 Le numéro 26 de la Revue internationale d'éducation - Sèvres est consacré à "L'évaluation des systèmes éducatifs ". Un résumé des articles du dossier est disponible en trois langues sur le site du CIEP : http://www.ciep.fr/ries/ries26.php ainsi que les références bibliographiques qui viennent en appui au dossier : http://www.ciep.fr/bibliographie/ ries26.php.

\section{Évaluations internationales : cadre général}

6 L'IEA (International association for the evaluation of educational achievement), organisme qui regroupe des représentants nationaux de différents systèmes éducatifs, fédère l'ensemble des évaluations par discipline. http://www.iea.nl/ Il offre sur son site des comptes rendus de modules d'utilisation des technologies dans l'enseignement primaire et secondaire dans plusieurs pays qui analysent la pratique de cet enseignement. Le site http://www.iea.nl/Home/body_home.html/ propose des liens vers les programmes d'évaluation.

$7 \quad$ L'International study center de Boston, centre de recherches qui vient en appui aux études menées par l'IEA, mène des études comparatives dans le domaine de l'évaluation en éducation. Le site offre des liens qui montrent de manière explicite la mise en place de la technique de benchmarking ${ }^{2}$, issue de la démarche qualité des entreprises, appliquée au domaine de l'éducation. Liens vers des pages consacrées à l'évaluation de l'éducation civique, de la lecture, de l'enseignement des sciences et des mathématiques pour les enfants entre 9 et 13 ans : http://timss.bc.edu/index.html. Le Boston College offre par ailleurs une nouvelle revue en ligne sur l'évaluation scolaire grâce aux technologies de l'information et de la communication, le «Journal of technology, learning and assesment $»$ :

8 http://www.bc.edu/research/intasc/jtla.html. Un article, « Automated Essay Scoring Using Bayes' Theorem » rend compte d'une étude qui applique à l'évaluation de textes littéraires les procédés de calculs de probabilités mis au point par le mathématicien Thomas Bayes : httpbc.edu/research/intasc/jtla/journal/v1n2.shtml

\section{Organismes européens}

9 Eurydice, réseau d'information sur l'éducation en Europe, a mis en ligne des rapports nationaux sur « les modes d'évaluation des établissements d'enseignement national ». Le rapport de chaque pays suit la même structure afin de permettre les comparaisons entre les pays. Chacun comporte une évaluation externe, une évaluation interne et une évaluation mixte. La terminologie employée est disponible en trois langues. http:// www.eurydice.org/Documents/Evaluation/fr/frameset_eval.html

Le réseau européen des responsables des politiques d'évaluation des systèmes éducatifs, organisme issu de la Commission européenne, regroupe des représentants des 15 États 
ainsi que l'Islande et la Norvège. Le site du réseau offre la possibilité de lire au format PDF leurs publications: actes de colloque, études, et lettre d'information. http:// www.isad.adc.education.fr/reva/france/publications.htm

\section{Associations}

11 Le site "educational testing service 》 fournit des informations concernant les tests d'évaluation et la recherche dans le domaine de l'évaluation: http://www.ets.org/ (USA)

12 European Educational Research Association: http://www.eera.ac.uk/. Recherche en éducation, conférences et événements: http://www.eera.ac.uk/events.html, publications http://www.eera.ac.uk/publications.html, sélection de sites Internet: http://www.eera.ac.uk/links.html. Instituts et associations; revues électroniques sur l'éducation ; systèmes éducatifs et sites institutionnels, programmes / éducation, etc.

13 ADMEE Europe: Association pour le développement des méthodologies d'évaluation en éducation: http://www.irdp.ch/admee. Cette association européenne offre en particulier sur son site des informations concernant le quinzième colloque international qu'elle a organisé en septembre 2002 sur « la qualité dans la formation et l'enseignement : comment la définir, comment l'évaluer?».

\section{Programmes d'évaluations internationales}

PISA (Programm for international students assessment) : l'étude, réalisée en 2000 dans 32 pays auprès de 250000 jeunes, a été conçue pour fournir des indications pertinentes sur les politiques éducatives, les connaissances et les savoir-faire des élèves âgés de 15 ans, en fin de scolarité obligatoire. L'évaluation a porté sur trois domaines : culture scientifique, culture mathématique et compréhension de l'écrit. Les résultats de cette étude ont été publiés à compter de la fin de l'année 2001 et sont disponibles sur la page du site de l'OCDE qui donne la liste des rapports nationaux de chaque pays participant à l'évaluation PISA 2000. http://www.pisa.oecd.org/NatReports/cntry.htm

PIRLS : PIRLS 2001 (Progress in international reading literacy study): évaluation de l'enseignement de la lecture dans l'enseignement primaire et secondaire: http:// www.imss.bc.edu/pirls2001.html

CIVED (Civic education study) : évaluation de l'éducation civique concernant des élèves de l'âge de 14 ans http://www2.hu-berlin.de/empir_bf/iea_e.html

SITES (Second information technology in education study): Module 1: http:// www.mscp.edte.utwente.nl/sitesm1/ Module 2: http://www.itesm2.org/ SITES_Research_Projects/SITESM2/sitesm2.html

TIMMS (Trends in international mathematics and science study): évaluation de l'enseignement scientifique avec possibilité de consulter aussi bien les programmes achevés que les programmes en cours : TIMMS 1995 : httpimss.bc.edu/timss1995.html. TIMMS 1999 : httpimss.bc.edu/timss1999.html. TIMSS 2003 : en cours httpimss.bc.edu/ timss2003.html. TIMSS-R Video. : http://www.lessonlab.com/research/timssr1.html. 


\section{Évaluation nationale : le cas de la France}

19 Nous avons choisi de ne traiter qu'un exemple national - celui de la France - en essayant dans ce cas de couvrir plusieurs champs de l'évaluation. Pour connaître les ressources d'autres pays, vous pouvez consulter le site suivant qui répertorie les adresses des gouvernements et de leurs ministères (classement par continent puis par pays) : http://www.gksoft.com/govt/en/.

LE HAUT CONSEIL DE L'EVALUATION DE L'ECOLE : http://www.isad.adc.education.fr/ hcee/default.htm. Présentation de l'instance française créée en novembre 2000, de ses missions et de ses membres: http://www.education.gouv.fr/ram/educd/brochure/ actions/2000/evaluation/hconseva.htm.

Le COMITE NATIONAL D’EVALUATION http://www.cne-evaluation.fr/, spécialisé dans l'enseignement supérieur, fournit des informations sur ce qu'est l'évaluation, son actualité, les programmes en cours et de nombreux liens vers d'autres sites, en particulier des sites sur l'évaluation institutionnelle dans le monde concernant l'enseignement supérieur: http://www.cne-evaluation.fr/fr/autresites/som_eva.htm . Les publications récentes sont disponibles au format PDF: http://www.cneevaluation.fr/fr/present/som_mis.htm

Site sur l'évaluation et les statistiques de l'éducation du MINISTERE DE L'EDUCATION NATIONALE français: http://www.education.gouv.fr/dpd//. Ce site est issu de la direction de la programmation et du développement (DPD). Il est régulièrement mis à jour. Outre une présentation $\mathrm{du}$ système éducatif français (http:// www.education.gouv.fr/dpd//DPD_WEB/index.htm), le site fournit les chiffres de l'Éducation nationale, des statistiques, un dossier sur l'évaluation $\mathrm{CE} / 6^{\mathrm{e}}\left(\mathrm{n}^{\circ} 128\right)$ : http://www.education.gouv.fr/dpd//dossiers/listedossiers.html et les résultats de ces évaluations : httpsg.education.gouv.fr/Evace26/debut.htm

Sur EDUSCOL, une partie du site est consacrée à l'évaluation en CE2-PPA (programmes personnalisés d'aide et de progrès). Au sommaire de la rubrique : finalités et usages des évaluations, mieux comprendre pour mieux agir, apporter des réponses, dispositifs d'accompagnement. Un espace échange est mis à disposition des internautes. http:// www.eduscol.education.fr/D0069/

Le BULLETIN OFFICIEL de l'Éducation nationale qui présente les outils nationaux de l'année 2002-2003, suivant les indications du Haut Conseil de l'évaluation, a pour but de différencier ceux qui constituent des évaluations bilan nationales: http:// www.education.gouv.fr/bo/2002/19/default.htm et ceux qui relèvent des évaluations diagnostiques de CE2, sixième et cinquième sur lesquelles nous reviendrons dans un numéro ultérieur.

\section{NOTES}

1. http://www.education.gouv.fr/discours/2001/innovation/rapport.htm\#evaluer. 
2. benchmarking: processus d'évaluation des services et produits d'une entreprise qui consiste à sélectionner les meilleures pratiques des concurrents afin de s'améliorer. http:// www.stratenet.com/benchmarking.html.

INDEX

Mots-clés : évaluation, politique éducative, système éducatif

\section{AUTEUR}

\section{FRANÇOISE PROFIT}

Documentaliste au Centre de ressources du CIEP 